\title{
Testing the sharing rule in a collective model of discrete labor supply with Spanish data
}

Jorge Velilla*

University of Zaragoza, Spain

Orcid: 0000-0002-0553-6360

\begin{abstract}
This paper estimates a collective model of discrete labor supply, using data from the Spanish Survey of Household Finances. The model allows identifying a sharing rule of household income. Then, it is used unique information for unemployed wives about intrahousehold transfers to estimate its accuracy. Results show that husbands' hours of work are conditional on wives' decisions, which mainly depend on non-labour income. Despite data availability, predicted sharing rules fit the data qualitatively well, and are mainly driven by wives' potential income. Husbands show low levels of altruism, and nonparticipation appears to be especially detrimental for wives with high potential income.
\end{abstract}

Keywords: Collective model; sharing rule; intrahousehold transfers; Spanish Survey of Household Finances

JEL codes: D13, J22

\footnotetext{
*Corresponding author: J. Velilla. Email: jvelilla@unizar.es.
} 


\section{Introduction}

For the implementation of family policies, such as welfare programs, it is important to understand the effects on intrahousehold decisions. Several models have been proposed to analyze intrahousehold behaviors, one being the collective model (Chiappori, 1988, 1992). The main hypothesis of such models is Pareto efficiency, i.e., spouses cooperate to take advantage of marriage. See, for instance, Donni and Chiappori (2011), or Donni and Molina (2018). The efficiency hypothesis of collective models allows identifying the theoretical sharing rule that should be characterized by spouses' (unobserved) bargaining powers. However, surveys rarely include the required information to study the accuracy of these sharing rules. So far, only Lise and Yamada (2018) and Bargain et al. (2018) have addressed this issue, using consumption data for Japan and Bangladesh, respectively.

In that context, this paper exploits information about intrahousehold transfers from employed to unemployed spouses in the Spanish Survey of Household Finances to study the accuracy of sharing rules derived from a collective model of discrete labor supply. Results point to the validity of the collective model, as the rational collectivity is not rejected. Furthermore, the marginal relationships between sharing rules and explanatory variables are consistent with reported intrahousehold transfers.

\section{The model}

The empirical study is based on the collective model of discrete labor supply of Blundell et al. (2007), adapted to female discrete labor participation, and male hours of work. ${ }^{1}$ Consider a household formed by a female $f$, and a male $m$, that reaches Pareto-efficient outcomes. Spouses have distinct egoistic utilities, $U_{i}=U_{i}\left(1-h^{i}, c_{i}\right), i=f, m$, where $h^{i}$ represents labor supply and $c_{i}$ private consumption. Define $w_{f}$ as $f$ 's labor income, $w_{m}$ as $m$ 's wage, and $y$ as household non-labor income. The household then solves:

$$
\max U_{f}+\mu U_{m}
$$

\footnotetext{
${ }^{1}$ This choice is intended to maximize the information available $(14.16 \%$ of husbands and $19.10 \%$ wives were unemployed in the original sample). Nevertheless, as shown in Table A1, the standard deviations of work hours are slightly larger for men than for women. Therefore, the assumption of a discrete female labor supply might not be accurate. Analogous main results for male discrete labor supply are shown in Table A3 in the Appendix.
} 


$$
\begin{aligned}
& \text { s.t. } C=w_{f} h^{f}+w_{m} h^{m}+y, \\
& h^{f} \in\{0,1\}, 0 \leq h^{m} \leq 1 .
\end{aligned}
$$

$\mu=\mu\left(w_{f}, w_{m}, y\right)$ is the Pareto weight, characterizing spouses' bargaining power.

Assume that $w_{f}$ represents potential earnings if $f$ does not work, and that wives' decisions are parametrized as:

$$
h^{f}=b_{0}+b_{1} w_{f}+b_{2} \log w_{m}+b_{3} y+b_{4} z
$$

where $z$ represents socio-demographics. Husbands' hours of work depend consequently on wives' decisions:

$$
h^{m}=\left\{\begin{array}{l}
A_{0}+A_{1} w_{f}+A_{2} \log w_{m}+A_{3} y+A_{4} z, \text { if } h^{f}=1, \\
a_{0}+a_{1} w_{f}+a_{2} \log w_{m}+a_{3} y+a_{4} z+, \text { if } h^{f}=0 .
\end{array}\right.
$$

In that context, the problem is identified if and only if observed behaviors satisfy a series of restrictions (e.g., the rational collectivity). Under Equations (7) and (9), the rational collectivity is:

$$
\frac{A_{1}-a_{1}}{A_{3}-a_{3}}=\frac{b_{1}}{b_{3}}, \frac{A_{2}-a_{2}}{A_{3}-a_{3}}=\frac{b_{2}}{b_{3}}
$$

If the data satisfies (4), the sharing rule of household income, $\phi$, can be identified up to an integration constant: ${ }^{2}$

$$
\begin{aligned}
& \phi_{w_{f}}=\frac{a_{1}}{\left(\frac{A_{1}}{A_{3}}-\frac{a_{1}}{a_{3}}\right) a_{3}}\left(\frac{A_{1}}{A_{3}}-1-\frac{A_{1}}{A_{3} F^{\prime}}\right), \\
& \phi_{w_{m}}=\frac{b_{2}}{\left(\frac{A_{1}}{A_{3}}-\frac{a_{1}}{a_{3}}\right) b_{3}}\left(\frac{A_{1}}{A_{3}}-1-\frac{a_{1}}{a_{3} F^{\prime}}\right), \\
& \phi_{y}=\frac{1}{\frac{A_{1}}{A_{3}}-\frac{a_{1}}{a_{3}}}\left(\frac{A_{1}}{A_{3}}-1-\frac{a_{1}}{a_{3} F^{\prime}}\right) .
\end{aligned}
$$

$2 F^{\prime}$ is a (positive) solution of $\left(F^{\prime}\right)^{2}\left(\frac{b_{3}}{b_{1}} \frac{a_{1}}{a_{3}}-\frac{b_{3}}{b_{1}} \frac{a_{1}}{a_{3}} \frac{A_{1}}{A_{3}}-1+\frac{A_{1}}{A_{3}}\right)+F^{\prime}\left(1-\frac{a_{1}}{a_{3}}+2 \frac{b_{3}}{b_{1}} \frac{a_{1}}{a_{3}} \frac{A_{1}}{A_{3}}-\frac{b_{3}}{b_{1}} \frac{A_{1}}{A_{3}}-\frac{A_{1}}{A_{3}}\right)+$ $\left(\frac{a_{1}}{a_{3}}-\frac{b_{3}}{b_{1}} \frac{a_{1}}{a_{3}} \frac{A_{1}}{A_{3}}\right)=0$. 


\section{Data}

I use the Survey of Household Finances (SHF) from years 2002-2014. The SHF is carried out every three years by the Bank of Spain and includes interviews at the family and individual level. I restrict the sample to spouses between 18 and 65 years, where the husband is employed, and the wife is either employed or not. Restrictions leave 4,170 households, $84.22 \%$ of which are characterized by both spouses working.

The following variables are used: age, education, marital status, the number of children, monthly earnings, weekly hours of work, hourly wages, and household non-labor income (household monthly expenses minus labor incomes). The SHF allows to define transfers from working to unemployed spouses. 101 of 658 unemployed wives report positive transfers but, as surveys rarely include direct information about intrahousehold processes (Bargain et al., 2018), it is worth examining these transfers. Table A1 in the Appendix shows main descriptive statistics.

Predicted $w_{f}, w_{m}$, and $y$ are defined to deal with potential endogeneity. The following Heckman models are estimated:

$$
\begin{aligned}
& w_{f}=\alpha_{0}+\alpha_{1} e d u_{f}+\alpha_{2} P^{2}\left(a g e_{f}\right)+\alpha_{4} z+\epsilon_{f}, \\
& \log w_{m}=\beta_{0}+\beta_{1} e d u_{m}+\beta_{2} P^{2}\left(a g e_{m}\right)+\beta_{4} z+\epsilon_{m},
\end{aligned}
$$

based on a human capital approach (Chiappori and Meghir, 2015). Non-labor income is instrumented as:

$$
y=\theta_{0}+\theta_{1} e d u_{f}+\theta_{2} e d u_{m}+\theta_{3} P\left(a g e_{m}, a g e_{f}\right)+\theta_{4} g(\mu)+\theta_{7} z+\epsilon,
$$

where $\mu$ represents household income from assets and dividends. All models include occupation and year fixed effects. Estimates are shown in Table A2 in the Appendix.

\section{Results}

Labor supply estimates are shown in Table 1. Column (1) shows that, on average, labor participation among wives is negatively determined by household income. On the other hand, wives' (husbands') earnings show a small, positive (negative) and non-significant correlation. 
Columns (2) and (3) show estimates for husbands whose wives work and do not work, respectively. Working wives' earnings are negatively correlated to husbands' hours of work. However, non-working wives' potential income is not significant. Analogously, husbands' wages are negative and significantly correlated with their labor supply only if the wife does not work. Finally, non-labor income shows a negative, small and significant correlation with husbands' hours of work.

(Table 1 about here)

Estimates do not reject the rational collectivity $(\mathrm{p}=0.894,0.431)$. Then, I can identify the sharing rule as:

$$
\phi=K-0.023 w_{f}+49.04 \log w_{m}+0.112 y .
$$

Thus, an increase in wives' potential earnings has a small and negative effect on their share of income. Oppositely, a 1\% increase in husband's wages (24.0 more Euros per month) is correlated with 4.9 Euros transferred to the wife; and from each extra Euro of non-labor income, 0.11 are assigned to wives. These results suggest moderate levels of altruism among husbands.

The mean difference between sharing rules and reported transfers is 117.95 Euros, which is non-null based on a t test $(p=0.018)$. Furthermore, $(8)$ is estimated using information about transfers in Column (4) of Table 1. Despite the existence of quantitative differences, results suggest that the sign of the derivatives of the sharing rule are accurate qualitatively.

\section{Conclusions}

This paper estimates a collective model of (discrete) labor supply using the Spanish Survey of Household Finances to study the accuracy of sharing rules. Results show that, despite the existence of quantitative differences, sharing rules are qualitatively robust to data. That provides evidence in favor of collective models.

The analysis has certain limitations. First, the data is cross-sectional. Second, sample restrictions may lead to selection bias. Finally, the information regarding transfers is limited to a reduced subsample. 


\section{Disclosure statement}

No potential conflict of interest was reported by the author.

\section{Acknowledgements}

Financial support provided by the Government of Aragón (Program FSE 2014-2020 and Project S32_17R) is gratefully acknowledged. Remaining errors are my exclusive responsibility.

\section{References}

Bargain, O., G. Lacroix and L. Tiberti. 2018. "Validating the Collective Model of Household Consumption Using Direct Evidence on Sharing.” IZA DP 11653.

Blundell, R., P.-A. Chiappori, T. Magnac and C. Meghi. 2007. "Collective labour supply: Heterogeneity and nonparticipation." Review of Economic Studies 74(259): 417-47.

Chiappori, P.-A. 1988. "Rational household labor supply.” Econometrica 56(1): 63-90.

Chiappori, P.-A. 1992. "Collective labor supply and welfare." Journal of Political Economy 100(3): 437-467.

Chiappori, P.-A. and C. Meghir. 2015. "Intrahousehold inequality." In Handbook of Income Distribution (Vol. 2, pp. 1369-1418). Elsevier.

Donni, O. and P.-A. Chiappori. 2011. "Non-unitary models of household behavior: A survey of the literature.” In Household Economic Behaviors, J. A. Molina (ed.). Berlin: Springer Verlag.

Donni, O. and J. A. Molina. 2018. "Household Collective Models: Three Decades of Theoretical Contributions and Empirical Evidence.” IZA DP 11915.

Lise, J. and K. Yamada. 2018. "Household sharing and commitment: Evidence from panel data on individual expenditures and time use." Review of Economic Studies, forthcoming. 
Table 1. Collective model estimates

\begin{tabular}{|c|c|c|c|}
\hline \multirow[b]{2}{*}{ VARIABLES } & \multirow{2}{*}{$\begin{array}{l}\text { Wives' labor } \\
\text { participation } \\
\text { (1) }\end{array}$} & \multicolumn{2}{|c|}{ Husbands' hours of work } \\
\hline & & $\begin{array}{c}\text { Wife works } \\
\text { (2) }\end{array}$ & $\begin{array}{c}\text { Wife not works } \\
\text { (3) }\end{array}$ \\
\hline Wife earnings & $\begin{array}{c}0.000 \\
(0.000)\end{array}$ & $\begin{array}{c}-0.003 * * * \\
(0.001)\end{array}$ & $\begin{array}{c}0.001 \\
(0.001)\end{array}$ \\
\hline Husband log-wage & $\begin{array}{l}-0.226 \\
(0.439)\end{array}$ & $\begin{array}{l}-1.928 \\
(1.511)\end{array}$ & $\begin{array}{c}-6.007 * * * \\
(1.107)\end{array}$ \\
\hline Non-labor income & $\begin{array}{c}-0.001 * * * \\
(0.000)\end{array}$ & $\begin{array}{c}-0.002 * * * \\
(0.001)\end{array}$ & $\begin{array}{c}-0.002 * * \\
(0.001)\end{array}$ \\
\hline Secondary (non-comp.) ed. (w) & $\begin{array}{c}0.211 \\
(0.148)\end{array}$ & - & - \\
\hline University ed. (w) & $\begin{array}{c}0.088 \\
(0.296)\end{array}$ & - & - \\
\hline Secondary (non-comp.) ed. (h) & - & $\begin{array}{c}-1.011 * * \\
(0.482)\end{array}$ & $\begin{array}{c}0.413 \\
(0.889)\end{array}$ \\
\hline University ed.(h) & - & $\begin{array}{c}-2.065^{* *} \\
(0.966)\end{array}$ & $\begin{array}{l}-2.275 \\
(1.825)\end{array}$ \\
\hline Age $(w)$ & $\begin{array}{l}-0.003 \\
(0.010)\end{array}$ & - & - \\
\hline Age (h) & - & $\begin{array}{l}-0.024 \\
(0.038)\end{array}$ & $\begin{array}{l}-0.056 \\
(0.053)\end{array}$ \\
\hline Married couple & $\begin{array}{c}0.296 \\
(0.181)\end{array}$ & $\begin{array}{l}-1.067 \\
(0.946)\end{array}$ & $\begin{array}{c}-5.194 * * * \\
(1.628)\end{array}$ \\
\hline N. children $\leq 6$ years & $\begin{array}{c}0.112 \\
(0.099)\end{array}$ & $\begin{array}{c}0.093 \\
(0.284)\end{array}$ & $\begin{array}{c}1.856^{* *} \\
(0.795)\end{array}$ \\
\hline N. children older & $\begin{array}{c}0.090 \\
(0.089)\end{array}$ & $\begin{array}{c}0.297 \\
(0.249)\end{array}$ & $\begin{array}{c}0.894 \\
(0.734)\end{array}$ \\
\hline Constant & $\begin{array}{c}1.016 \\
(0.679)\end{array}$ & $\begin{array}{c}48.526 * * * \\
(2.103)\end{array}$ & $\begin{array}{c}58.063 * * * \\
(3.118)\end{array}$ \\
\hline $\begin{array}{l}\text { Year F.E. } \\
\text { Observations }\end{array}$ & $\begin{array}{c}\text { Yes } \\
4,170\end{array}$ & $\begin{array}{c}\text { Yes } \\
3,512\end{array}$ & $\begin{array}{l}\text { Yes } \\
658\end{array}$ \\
\hline
\end{tabular}

Note: Robust standard errors in parentheses. Estimates include sample weights. $* * *$ Significant at the $1 \%$, ** significant at the $5 \%, *$ significant at the $10 \%$. 


\section{Appendix A: Additional tables}

Table A1. Summary statistics

\begin{tabular}{lcccc}
\hline \multirow{2}{*}{ INDIVIDUAL VARIABLES } & \multicolumn{2}{c}{ Wives } & \multicolumn{2}{c}{ Husbands } \\
Labor participation & Mean & S.D. & Mean & S.D. \\
\cline { 2 - 5 } Hours of work per week $^{\dagger}$ & 0.842 & 0.365 & - & - \\
Monthly income $^{\dagger}$ & 29.728 & 15.613 & 41.838 & 8.451 \\
Monthly transfer $^{\dagger \dagger}$ & 1605.5 & 1244.7 & 2320.6 & 2504.7 \\
Hourly wage & 624.67 & 486.96 & - & - \\
& & & 13.401 & 15.924
\end{tabular}

Household

\begin{tabular}{lcc} 
FAMILY VARIABLES & Mean & S.D. \\
\cline { 2 - 3 } Monthly non-labor income & -2219.9 & 2801.9
\end{tabular}

N. Families 4,170

Note: Statistics include sample weights. ${ }^{\dagger}$ Sample restricted to employed wives. ${ }^{\dagger}$ Sample restricted to unemployed wives reporting non-null values. 
Table A2. First stage estimates

\begin{tabular}{|c|c|c|c|c|c|}
\hline \multirow[b]{2}{*}{ VARIABLES } & \multicolumn{2}{|c|}{ Wives' earnings } & \multicolumn{2}{|c|}{ Husbands' wages } & \multirow{2}{*}{$\begin{array}{l}\text { Non-labor } \\
\text { income } \\
\text { (5) }\end{array}$} \\
\hline & $\begin{array}{l}\text { Main eq. } \\
\text { (1) }\end{array}$ & $\begin{array}{l}\text { Selection eq. } \\
\text { (2) }\end{array}$ & $\begin{array}{l}\text { Main eq. } \\
\text { (3) }\end{array}$ & $\begin{array}{c}\text { Selection eq. } \\
\text { (4) }\end{array}$ & \\
\hline Secondary (non-comp.) ed. (w) & $\begin{array}{c}64.700 * * \\
(29.274)\end{array}$ & - & - & - & $\begin{array}{c}-128.376^{* *} \\
(59.637)\end{array}$ \\
\hline University ed. (w) & $\begin{array}{c}466.984 * * * \\
(55.638)\end{array}$ & - & - & - & $\begin{array}{c}-333.457^{* *} \\
(141.078)\end{array}$ \\
\hline Secondary (non-comp.) ed. (h) & - & - & $\begin{array}{c}0.110 * * * \\
(0.016)\end{array}$ & - & $\begin{array}{l}-82.051 \\
(63.939)\end{array}$ \\
\hline University ed. (h) & - & - & $\begin{array}{c}0.309^{* * *} \\
(0.024)\end{array}$ & - & $\begin{array}{c}- \\
498.362 * * * \\
(147.648)\end{array}$ \\
\hline Age (w) & $\begin{array}{c}36.656^{* * * *} \\
(11.989)\end{array}$ & - & - & - & $\begin{array}{c}15.210 \\
(33.893)\end{array}$ \\
\hline Age sq. (w) & $\begin{array}{l}-31.241^{*} \\
(16.479)\end{array}$ & - & - & - & $\begin{array}{l}-26.763 \\
(48.010)\end{array}$ \\
\hline Age (h) & - & - & $\begin{array}{c}0.009 \\
(0.006)\end{array}$ & - & $\begin{array}{l}-62.546^{*} \\
(37.468)\end{array}$ \\
\hline Age sq. (h) & - & - & $\begin{array}{l}-0.002 \\
(0.008)\end{array}$ & - & $\begin{array}{c}98.462 * * \\
(45.919)\end{array}$ \\
\hline Married couple & - & $\begin{array}{c}0.181 * * * \\
(0.060)\end{array}$ & - & $\begin{array}{c}0.329 * * * \\
(0.063)\end{array}$ & $\begin{array}{c}- \\
300.181 * * * \\
(82.052)\end{array}$ \\
\hline N. children $\leq 6$ years & - & $\begin{array}{l}-0.019 \\
(0.027)\end{array}$ & - & $\begin{array}{l}-0.007 \\
(0.031)\end{array}$ & $\begin{array}{c}139.324 * * \\
(63.403)\end{array}$ \\
\hline N. children older & - & $\begin{array}{c}0.071^{* * *} * \\
(0.025)\end{array}$ & - & $\begin{array}{c}0.003 \\
(0.027)\end{array}$ & $\begin{array}{c}127.962 * * * \\
(48.889)\end{array}$ \\
\hline Main house: owned & $\begin{array}{c}110.491 * * * \\
(39.496)\end{array}$ & - & $\begin{array}{c}0.121 * * * \\
(0.019)\end{array}$ & - & $\begin{array}{c}-74.448 \\
(142.616)\end{array}$ \\
\hline N. other houses & $\begin{array}{c}67.773 * * * \\
(9.897)\end{array}$ & - & $\begin{array}{c}0.029 * * * \\
(0.008)\end{array}$ & - & $\begin{array}{c}- \\
307.372 * * * \\
(101.908)\end{array}$ \\
\hline N. vehicles & $\begin{array}{c}26.367 \\
(31.054)\end{array}$ & - & $\begin{array}{c}0.057 * * * \\
(0.013)\end{array}$ & - & $\begin{array}{c}35.862 \\
(65.946)\end{array}$ \\
\hline Monthly income from assets & - & - & - & - & $\begin{array}{c}0.695 * * * \\
(0.243)\end{array}$ \\
\hline Monthly income from assets sq. & - & - & - & - & $\begin{array}{l}0.041^{* *} \\
(0.019)\end{array}$ \\
\hline Positive assets & - & - & - & - & $\begin{array}{c}- \\
404.859^{* * *} \\
(67.881)\end{array}$ \\
\hline Positive assets* & & & & & \\
\hline Monthly income from assets & - & - & - & - & $\begin{array}{c}-1.353 * * * \\
(0.520)\end{array}$ \\
\hline Monthly income from assets sq. & - & - & - & - & $\begin{array}{c}-0.041 * * \\
(0.019)\end{array}$ \\
\hline Constant & $\begin{array}{c}867.688 * * * \\
(240.453)\end{array}$ & $\begin{array}{c}0.696^{* * *} * \\
(0.057)\end{array}$ & $\begin{array}{c}1.937 * * * \\
(0.118)\end{array}$ & $\begin{array}{c}0.812 * * * \\
(0.059)\end{array}$ & $\begin{array}{l}-1,492 * * \\
(624.469)\end{array}$ \\
\hline Inverse Mills ratio & $\begin{array}{c}-9.769 \\
(36.192)\end{array}$ & - & $\begin{array}{l}-0.068 \\
(0.044)\end{array}$ & - & - \\
\hline Occupation F.E. & Yes & Yes & Yes & Yes & Yes \\
\hline Year F.E. & Yes & Yes & Yes & Yes & Yes \\
\hline Observations & 4,856 & 4,856 & 4,856 & 4,856 & 4,853 \\
\hline
\end{tabular}

Note: Robust standard errors in parentheses. Estimates include sample weights. $* * *$ Significant at the $1 \%, * *$ significant at the $5 \%,{ }^{*}$ significant at the $10 \%$. 
Table A3. Collective model estimates - discrete male labor supply

\begin{tabular}{|c|c|c|c|c|}
\hline \multirow[b]{2}{*}{ VARIABLES } & \multirow{2}{*}{$\begin{array}{c}\text { Husbands' labor } \\
\text { participation } \\
\text { (1) }\end{array}$} & \multicolumn{2}{|c|}{ Wives' hours of work } & \multirow{2}{*}{$\begin{array}{c}\text { Estimated } \\
\text { Sharing rule } \\
\text { (4) }\end{array}$} \\
\hline & & $\begin{array}{l}\text { Husband works } \\
\text { (2) }\end{array}$ & $\begin{array}{c}\text { Husband does } \\
\text { not work } \\
\text { (3) }\end{array}$ & \\
\hline Husband earnings & $\begin{array}{l}-0.000 \\
(0.000)\end{array}$ & $\begin{array}{l}-0.001 \\
(0.000)\end{array}$ & $\begin{array}{l}-0.000 \\
(0.001)\end{array}$ & $\begin{array}{l}-0.160 \\
(0.161)\end{array}$ \\
\hline Wife log-wage & $\begin{array}{l}0.735^{*} \\
(0.439)\end{array}$ & $\begin{array}{c}5.659 * * * \\
(1.326)\end{array}$ & $\begin{array}{c}-7.561 * * * \\
(2.039)\end{array}$ & $\begin{array}{c}372.182 * * * \\
(112.428)\end{array}$ \\
\hline Non-labor income & $\begin{array}{c}-0.000 * * * \\
(0.000)\end{array}$ & $\begin{array}{l}-0.000 \\
(0.001)\end{array}$ & $\begin{array}{c}-0.004 * * * \\
(0.001)\end{array}$ & $\begin{array}{c}-0.213^{* *} \\
(0.101)\end{array}$ \\
\hline Constant & $\begin{array}{c}1.862 * * * \\
(0.621)\end{array}$ & $\begin{array}{c}30.613 * * * \\
(2.014)\end{array}$ & $\begin{array}{c}49.162 * * * \\
(4.295)\end{array}$ & - \\
\hline Socio-demographics & Yes & Yes & Yes & No \\
\hline Observations & 3,943 & 3,512 & 431 & 36 \\
\hline
\end{tabular}

Note: Robust standard errors in parentheses. Estimates include sample weights. *** Significant at the $1 \%$, ** significant at the $5 \%, *$ significant at the $10 \%$. 